\title{
Contrasting the grain boundary-affected performance of zinc and indium oxide transparent conductors
}

\author{
A T Vai, N Rashidi, Y Fang, V L Kuznetsov and P P Edwards* \\ Inorganic Chemistry Laboratory, Department of Chemistry, University of Oxford, South \\ Parks Road, OXFORD OX1 3QR UK \\ *E-mail: peter.edwards@ chem.ox.ac.uk Fax: +44 (0)1865 272656
}

\begin{abstract}
Zinc oxide-based transparent conductors have long been advanced for their potential as low-cost, earth-abundant replacements for the indium oxide-based materials that currently dominate in practical applications. However, this has yet to be realized because of the difficulties in producing zinc oxide thin films with the necessary high levels of electrical conductivity and environmental stability that are readily achieved using indium oxide. To better understand the fundamental reasons for this, polycrystalline zinc and indium oxide thin films were prepared across a range of deposition temperatures using the technique of spray pyrolysis. Electrical transport measurements of these samples both as a function of temperature and UV irradiation were correlated with film morphology to illustrate that the different grain boundary behaviour of these two materials is one of the key reasons for their divergent performance and a critical challenge that must be addressed before any successful adoption of $\mathrm{ZnO}$-based transparent conductors can take place.
\end{abstract}

PACS: $81.05 . \mathrm{Dz}$, 81.10.Dn, 71.20.Nr, 71.55.Gs, 72.10.Fk

Submitted to Journal of Physics: Condensed Matter

\section{Introduction}

Among the transparent conducting oxides (TCOs), materials based on indium oxide $\left(\operatorname{In}_{2} \mathrm{O}_{3}\right)$-most notably tin-doped indium oxide, or ITO — stand out for their extremely high and environmentally stable thin film electrical conductivities and for the relative ease with which this class-leading performance can be achieved on large scales in industrial settings worldwide.[1,2] Much is made of the fact that indium oxide is several orders of magnitude scarcer and more expensive than potential TCO competitors like zinc oxide $(\mathrm{ZnO})$. However, the simple fact that the technical performance of indium oxide has not yet been reliably matched by that of an alternative makes the economic argument more or less moot for end users, especially in the many TCO applications where high electrical conductivity is of paramount importance.

Specifically, it has proven challenging to produce doped $\mathrm{ZnO}$ thin films with electrical conductivity matching that which is readily achieved by ITO.[3] And even when relatively high conductivities in $\mathrm{ZnO}$ have been successfully achieved under careful laboratory conditions, the long-term stability of this performance to the levels of heat and humidity commonly used in lifespan testing of consumer electronics remains problematic.[4-7] 


\section{Grain boundary-affected performance of $\mathrm{Zn}$ and In oxide transparent conductors}

Obviously, a low-cost, earth-abundant alternative to ITO with comparable optoelectronic properties and durability would be highly useful and attractive. Indeed, the desire to find such a material has been one of the primary motivators behind a truly vast body of research into reduced indium content and indium-free TCO materials. Broadly speaking, the predominant approach has been to treat this as a combinatorial materials discovery problem, to be solved by searching over a composition space with concurrent empirical optimization of deposition parameters. For instance, even with the scope limited to just ZnO-based materials, there are reports of $\mathrm{ZnO}$ doped with one or more chemical elements from every region of the periodic table, except for the noble gases. When one further considers how sensitive the optoelectronic properties of a TCO thin film can be to the deposition technique employed and the exact choice of process parameters used within a specific implementation of that technique, the result is substantial scatter in reported material properties and great difficulty in deriving general conclusions about the status and prospects of the field from the results of any given study.[8]

Rather than contributing further to this already extensive mapping between deposition conditions and optoelectronic properties, our intention here is to perform a more fundamental comparative study between indium oxide and zinc oxide, with the goal of highlighting key differences between these materials. In this way, we hope to describe some of the physical processes that have so far most hindered the development of ITO alternatives, and thus point out areas that may be most productively targeted by future research.

Spray pyrolysis has been chosen as the primary technique for preparing zinc and indium oxide thin film samples in this study. Using this solution-based, atmospheric pressure technique, thin film TCO samples with a wide range of chemical compositions can be rapidly and flexibly prepared simply by using different precursor solutions.[9] This contrasts with many experimental setups for physical deposition (i.e., magnetron sputtering or pulsed-laser deposition) where the relatively time-consuming process of ceramic target preparation must be repeated for each set of compositions to be studied. Despite a reputation for being somewhat "less rigorous" than its vacuum-based counterparts, we have previously shown that with appropriate process controls and design, spray pyrolysis can be used to prepare samples with good enough reproducibility to allow for detailed examination of phenomena such as the doping dependent metal-insulator transition, and the evaluation of grain boundary effects as the root cause of a UV-induced enhancement in carrier mobility.[10, 11] 


\section{Grain boundary-affected performance of $\mathrm{Zn}$ and In oxide transparent conductors}

In the present study, the structural and electronic transport properties of zinc and indium oxide thin films deposited at various temperatures were characterized in detail. The temperature-dependent carrier mobilities of the samples were measured and modelled to interrogate and quantify the influence of potential barriers at grain boundaries on electronic transport in these polycrystalline samples. We have also used the persistent photoconductive response of these materials as a new type of probe to determine the extent to which conductivity in various samples is limited by the adsorption of species from the air at grain boundaries. These results are correlated here with film morphology and used to explain the divergent behaviour of $\operatorname{In}_{2} \mathrm{O}_{3}$ and $\mathrm{ZnO}$ deposited under these conditions, and in particular, the observation that $\operatorname{In}_{2} \mathrm{O}_{3}$ thin films are both more conductive and also more insensitive to deposition temperature than $\mathrm{ZnO}$ thin films. Our conclusions reinforce and extend those drawn by several prior studies in this field that grain boundary effects can have a critical, and indeed, a dominating influence on the macroscopic electrical performance of polycrystalline TCO materials, especially $\mathrm{ZnO} .[12,13]$

\section{Experimental methods}

\subsection{Film deposition}

The general apparatus and process used for the spray pyrolysis deposition of thin film TCO samples is as previously described.[10] Nitrogen was used as the atomization and carrier gas for all samples prepared in this study. Reported deposition temperatures $\left(T_{d e p}\right)$ are of the substrate surface based on measurements using an optical pyrometer. Temperature uniformity across the substrate surface is estimated to be $\pm 7^{\circ} \mathrm{C}$.

For the deposition of $\mathrm{ZnO}$, a $0.225 \mathrm{M}$ solution of anhydrous zinc acetate (99.99\% metals basis, Aldrich) is dissolved in a 7:93 (by volume) mixture of glacial acetic acid (Analytical Reagent Grade, Fisher Scientific) and deionized water (Ondeo Purite Select Analyst). For each standard deposition run, $4 \mathrm{~mL}$ of this aqueous solution is vigorously stirred with ethanol ( $8 \mathrm{~mL}, 99.8 \%$, puriss., absolute, Sigma-Aldrich). $10 \mathrm{~mL}$ of the latter mixture is used for each standard deposition. Since sample thickness is approximately proportional to the amount of precursor solution sprayed, a larger volume was used to prepare thicker samples.

For the deposition of $\operatorname{In}_{2} \mathrm{O}_{3}$, a precursor solution based on one previously described in the literature is used.[14] A $0.025 \mathrm{M}$ solution of indium (III) chloride hydrate (99.99\% metals basis, Alfa Aesar) is prepared in a 3:1 (by volume) mixture of $0.0375 \mathrm{M}$ hydrochloric acid (Analytical Regent Grade, Fisher Scientific) and ethanol. For each standard deposition, $50 \mathrm{~mL}$ of this solution is sprayed. 
Grain boundary-affected performance of $\mathrm{Zn}$ and In oxide transparent conductors

\subsection{General film characterization}

The envelope method of Swanepoel was used to estimate film thicknesses from the interference fringes in the high transmission portion of UV-Vis-NIR transmission spectra collected using a Perkin-Elmer Lambda19 spectrophotometer.[15] Except where otherwise noted, the $\mathrm{ZnO}$ samples used in this study were $\sim 400 \mathrm{~nm}$ thick for the samples deposited at $417{ }^{\circ} \mathrm{C}$ and $376{ }^{\circ} \mathrm{C}$, and were $\sim 250-300 \mathrm{~nm}$ for the samples deposited at lower temperatures. The thicknesses of the indium oxide samples also varied with deposition temperature and were from $250-400 \mathrm{~nm}$ thick, except for the samples deposited at $250{ }^{\circ} \mathrm{C}$ which were $\sim 150$ nm thick.

Hall effect measurements were performed with the van der Pauw method using an Ecopia HMS-3000 Hall effect measurement system with a $0.55 \mathrm{~T}$ permanent magnet. Indium solder was used to create four Ohmic electrical contacts on the corners of an approximately $7 \mathrm{~mm} \times 7 \mathrm{~mm}$ piece of the thin film sample. For samples deposited at lower heater temperatures that were not of sufficient conductivity to allow reliable carrier concentration and mobility measurements, only electrical resistivity is reported. Where shown, error bars indicate the standard deviation of a measured property across a population of samples prepared using the same nominal conditions and procedures.

Hall effect measurements at temperatures below (to $\sim 80 \mathrm{~K}$ ) and above (to $\sim 360 \mathrm{~K}$ ) ambient were achieved using a custom-designed heating and cooling system. Sample temperature was measured with a Type $\mathrm{T}$ thermocouple embedded in the sample mount and in contact with the film substrate. For all Hall Effect measurements, samples were allowed to equilibrate in the dark under open air prior to measurement.

The surface morphology of the thin films was characterized using a JEOL JSM-840F field emission scanning electron microscope at an accelerating voltage of $5 \mathrm{kV}$ and using a secondary electron detector. Samples were sputter coated with a $\sim 2.5 \mathrm{~nm}$ layer of platinum before observation in the SEM to reduce the effect of surface charging and attendant artefacts.

X-ray diffraction measurement of samples was performed using PANalytical X'Pert PRO diffractometers with Bragg-Brentano geometries. Fixed X-ray sources with an emission current of $40 \mathrm{~mA}$ and an anode voltage of $45 \mathrm{kV}$ were used. The spectra were recorded using either monochromated $\mathrm{Cu}-\mathrm{K}_{\alpha 1}(\lambda=$ $1.5406 \AA)$ or $\mathrm{Cu}-\mathrm{K}_{\alpha}(\lambda=1.5418 \AA)$ radiation, depending on the spectrometer used. Samples were placed on a $60 \mathrm{rpm}$ spinner to improve averaging of crystallite orientation. 


\section{Grain boundary-affected performance of $\mathrm{Zn}$ and In oxide transparent conductors}

\subsection{Photoconductivity measurements}

Approximately $10 \times 2 \mathrm{~mm}$ rectangular pieces of thin film were cut from larger deposited samples. Indium solder was used to create four electrical contacts along the long axis of the samples in an I+, V+, V-, Iconfiguration. Under air, the samples were allowed to equilibrate in the dark for several hours before being exposed to $365 \mathrm{~nm}$ centred radiation from a blacklight lamp (8W, UVP EL Series). The samples were illuminated at normal incidence from a distance of $\sim 10 \mathrm{~cm}$. Operations were carried out in a dark box that excluded other sources of light. Changes in the electrical resistance of samples were measured and recorded using an Agilent 34401A multimeter in 4-lead resistance mode.

\section{Results and discussion}

\subsection{Room-temperature electrical properties and morphology}

An examination of the electrical conductivity of nominally undoped $\operatorname{In}_{2} \mathrm{O}_{3}$ and $\mathrm{ZnO}$ thin films as a function of deposition temperature (Figure 1) shows two major qualitative differences between the materials. First, the average electrical conductivity of $\operatorname{In}_{2} \mathrm{O}_{3}$ samples deposited at each temperature is more than 10 times higher than that of the corresponding $\mathrm{ZnO}$ films. Second, the electrical conductivity of the $\operatorname{In}_{2} \mathrm{O}_{3}$ thin film samples remains largely constant across the entire spray pyrolysis deposition temperature window. This window is bounded on the low temperature side by incomplete decomposition of the indium chloride precursor, which can be seen from the signals corresponding to indium oxide chloride that appear in the X-ray diffraction patterns (Figure 2a) for the film deposited at $250{ }^{\circ} \mathrm{C}$ (as an impurity) and for the film deposited at $209{ }^{\circ} \mathrm{C}$ (as the majority phase). In other words, high conductivity is maintained as long as the deposited film is predominantly indium oxide. The same is clearly not the case for the $\mathrm{ZnO}$ thin film samples, whose sharp decline in conductivity as $T_{\text {dep }}$ decreases begins well above the threshold where any incomplete decomposition of the zinc acetate precursor can be observed (Figure $2 b$ ).

These two observations illustrate starkly some of the major challenges faced in trying to replace indium-oxide based materials with zinc oxides, which we stress are present whether the chosen deposition routes are solution-based or high-vacuum. Specifically, even under the relatively relaxed environmental controls of spray pyrolysis (i.e. aqueous solvents, non-hermetic deposition chambers), $\operatorname{In}_{2} \mathrm{O}_{3}$ and ITO films with electrical conductivity exceeding $10000(\Omega \mathrm{cm})^{-1}$ can be straightforwardly prepared, meeting or exceeding the conductivity of commercial magnetron sputtered samples.[14] On top of that, the strong dependence of $\mathrm{ZnO}$ performance on deposition temperature (and as will be seen, other environmental influences) is also of 


\section{Grain boundary-affected performance of $\mathrm{Zn}$ and In oxide transparent conductors}

significant practical importance. Large area, flexible electronics are one of the main growth areas in TCO applications, and the strong correlation between high deposition temperature and performance has thus far made $\mathrm{ZnO}$-based materials incompatible with the temperature-sensitive polymers required for such uses.[16]

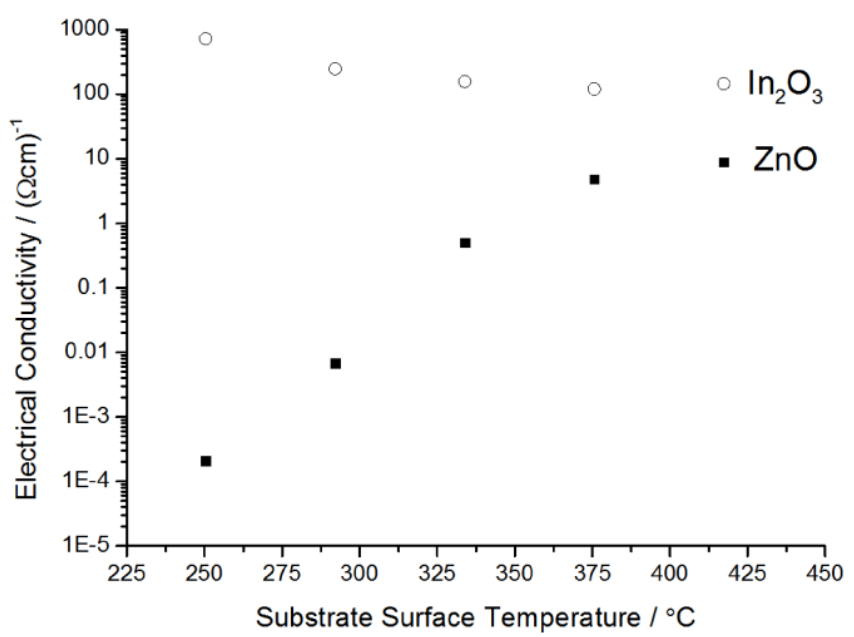

Figure 1. Electrical conductivity of undoped $\mathrm{In}_{2} \mathrm{O}_{3}$ (open circles) and $\mathrm{ZnO}$ (filled squares) thin films deposited by spray pyrolysis at various substrate temperatures. Data for samples prepared at $209^{\circ} \mathrm{C}$ is not shown because neither sample deposited at this temperature was measurably conductive.

a)

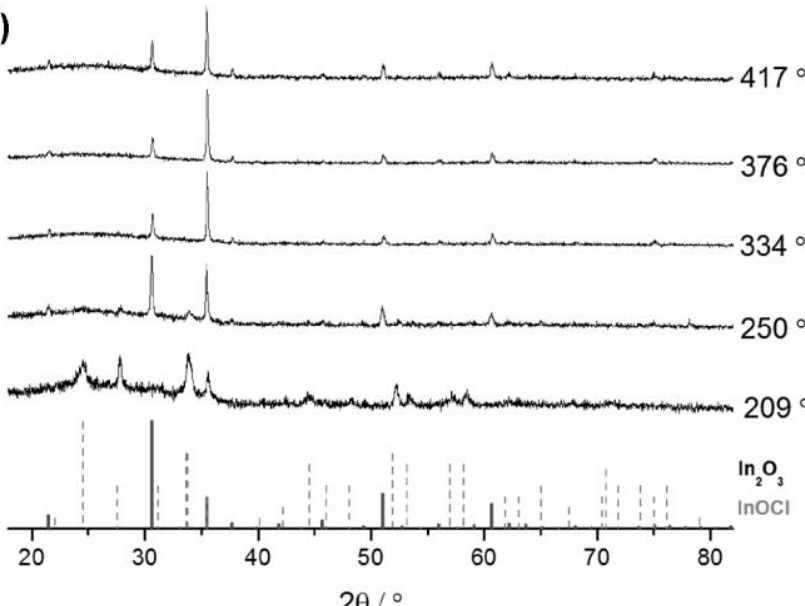

$2 \theta / \circ$

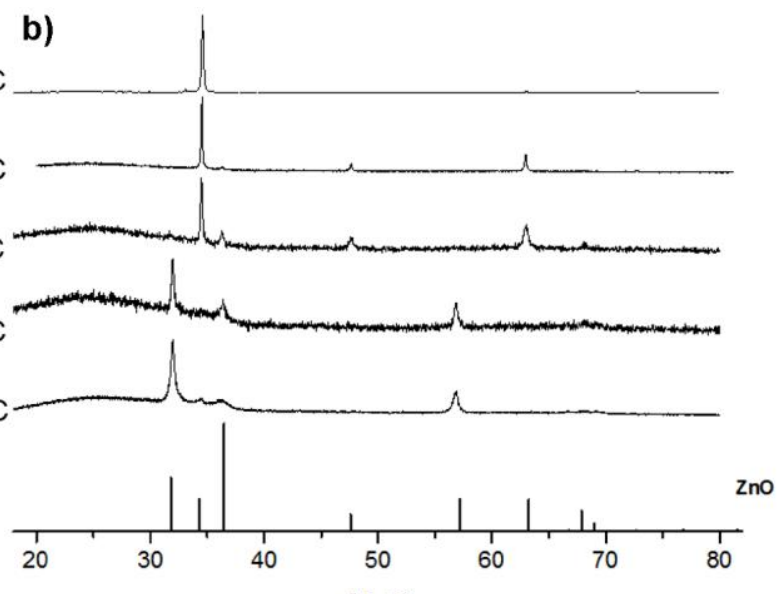

$2 \theta /^{\circ}$

Figure 2. X-ray diffraction patterns of (a) indium oxide and (b) zinc oxide thin films deposited by spray pyrolysis at various substrate temperatures. Note that (a) show incomplete decomposition of the indium chloride precursor (forming indium oxide chloride) in the samples at $250{ }^{\circ} \mathrm{C}$ and $209^{\circ} \mathrm{C}$. Reference patterns for $\operatorname{In}_{2} \mathrm{O}_{3}$ (a, solid lines), $\mathrm{InOCl}$ (a, dashed gray lines), and $\mathrm{ZnO}$ (b) are provided at the bottom of the figures.

The sharp decrease in electrical conductivity of the $\mathrm{ZnO}$ samples with decreasing deposition temperature is largely due to a decrease in Hall carrier mobility, while the carrier concentration stays roughly constant with deposition temperature (Figure 3). The $\mathrm{ZnO}$ samples deposited at $250{ }^{\circ} \mathrm{C}$ and $209{ }^{\circ} \mathrm{C}$ were too resistive for reliable Hall effect measurements, likely due to even lower carrier mobilities $\left(<<1 \mathrm{~cm}^{2} / \mathrm{Vs}\right)$ resulting at these deposition temperatures.

The carrier mobility of the $\mathrm{In}_{2} \mathrm{O}_{3}$ samples deposited at $\geq 292{ }^{\circ} \mathrm{C}$ is relatively constant (to within experimental error) around $40-50 \mathrm{~cm}^{2} / \mathrm{Vs}$, while there is a modest increase in the carrier concentration values 


\section{Grain boundary-affected performance of $\mathrm{Zn}$ and In oxide transparent conductors}

of the films as the deposition temperature decreases (Figure 3). However, these values do not vary by more than $\sim 50 \%$ in the range of $T_{\text {dep }}$, leading to the relatively constant electrical conductivity observed. $\operatorname{The} \operatorname{In}_{2} \mathrm{O}_{3}$ samples deposited at $250{ }^{\circ} \mathrm{C}$ exhibit markedly different values of carrier concentration and mobility from the rest of the series. This may be due to doping from residual chloride left in the film at these lower deposition temperatures, as evidenced by the small amount of indium oxide chloride impurity that can be clearly observed in the XRD patterns collected from this sample (Figure 2a).

a)

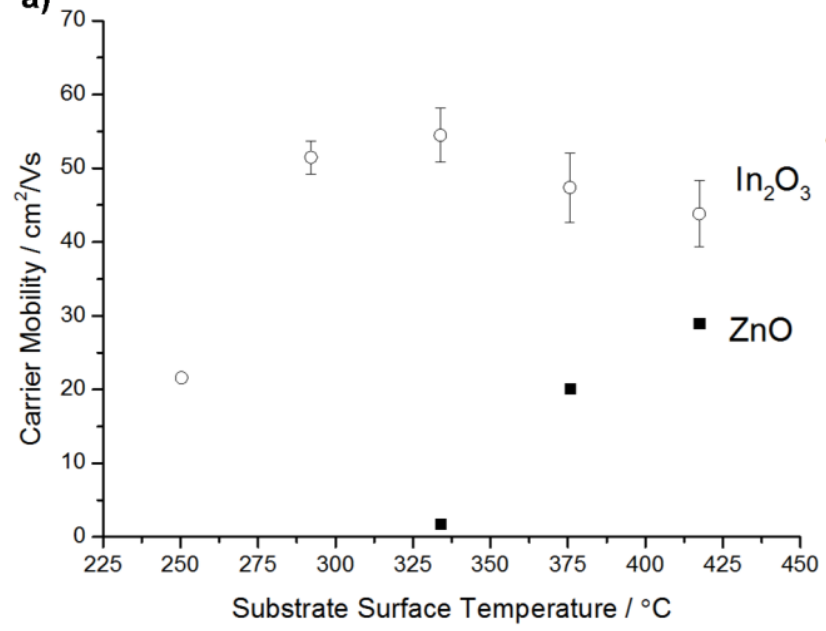

b)

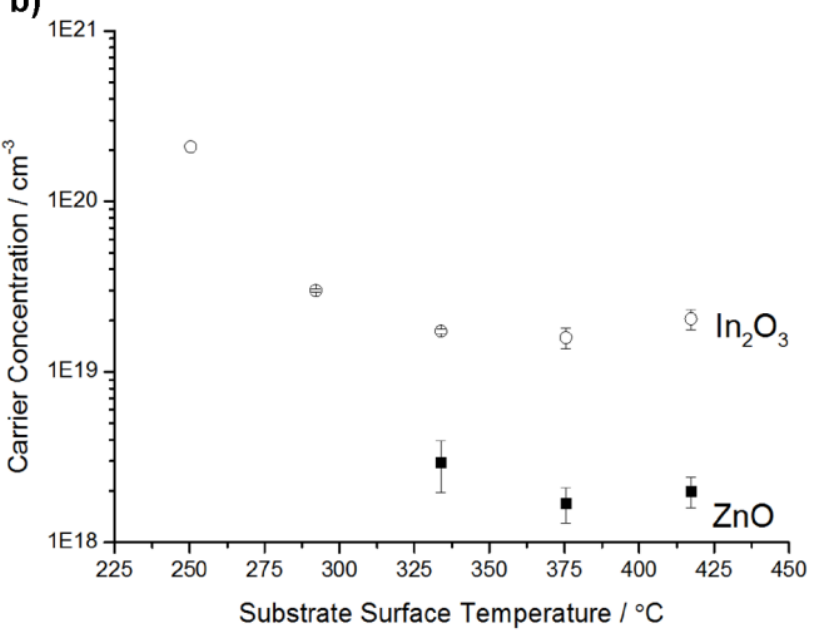

Figure 3. Hall effect (a) carrier mobility and (b) carrier concentration for indium oxide (open circles) and zinc oxide (filled squares) thin films deposited by spray pyrolysis at various substrate temperatures. Data is not shown for lower temperature zinc oxide samples because these samples were not conductive enough for reliable Hall effect measurements.

The fact that both the indium oxide and zinc oxide samples are polycrystalline, as is the case with the vast majority of TCO samples deposited by both vacuum and solution deposition methods, can be clearly observed from the XRD patterns of the two series (Figure 2) along with SEM images of the film surfaces (Figure 4). It should also be noted that both sets of XRD data show evidence for temperature-dependent preferred orientation of crystallites within these samples. When considering the electrical properties of these series, it is extremely interesting to note that carefully prepared single crystals of undoped, low carrier concentration $\mathrm{ZnO}$ and $\mathrm{In}_{2} \mathrm{O}_{3}$ are reported to have very similar carrier mobilities, and hence electrical conductivities.[17, 18] This strongly suggests that polycrystallinity itself is an important factor in explaining why $\mathrm{ZnO}$ and $\mathrm{In}_{2} \mathrm{O}_{3}$ have different electrical properties when deposited as thin films. 
Grain boundary-affected performance of $\mathrm{Zn}$ and In oxide transparent conductors

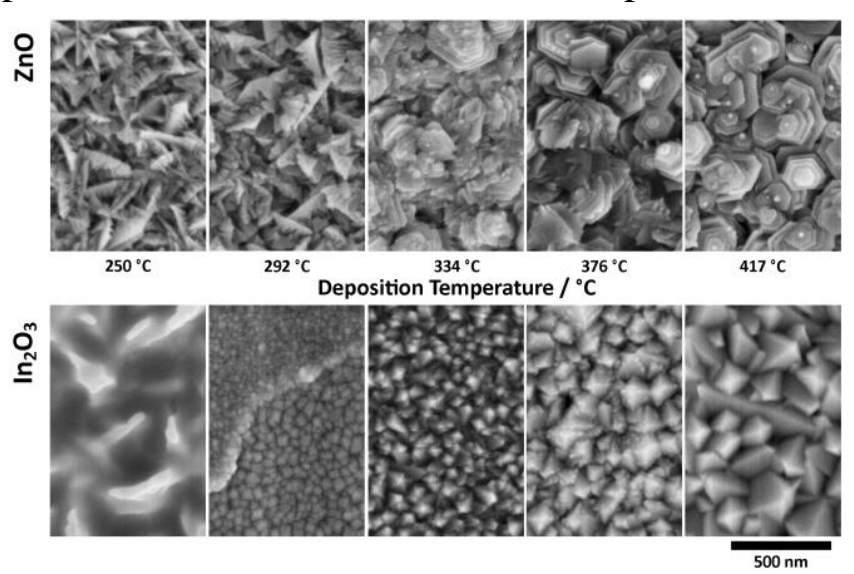

Figure 4. Representative SEM micrographs showing changes in the surface morphology of zinc oxide (top row) and indium oxide (bottom row) thin films deposited by spray pyrolysis as a function of deposition temperature.

\subsection{Temperature-dependent carrier mobility}

The defects at $\mathrm{ZnO}$ grain boundaries often lead to a preponderance of acceptor states, which are capable of trapping conduction electrons from nearby grains.[19] This localization of negative charge at the grain boundaries and the corresponding depletion of negative charge from nearby grain interiors leads to the formation of a potential barrier that reduces the inter-grain carrier mobility and is commonly modelled as a back-to-back Schottky barrier under a small bias.[20] Using this model, and considering only the effect of thermionic transport over potential barriers, Seto calculated that the grain boundary-limited mobility of nondegenerately conducting polycrystalline samples as:

$$
\mu_{g b}(T)=L e\left(\frac{1}{2 \pi m^{*} k_{B} T}\right)^{1 / 2} \exp \left(-\frac{V_{b}}{k_{B} T}\right),
$$

where $L$ is described as an "effective grain size", $e$ is the electronic charge, $m$ * is the carrier electron effective mass, $V_{b}$ is an activation energy related to the mean grain boundary barrier height, and $k_{B}$ is Boltzmann's constant.[21]

In order to account for the experimentally observed decrease in carrier mobility at higher temperatures, the effect of electron-phonon scattering on mobility also needs to be considered (Figure 5). In the temperature range under study, and assuming reasonable, literature values of $\mathrm{ZnO}$ and $\operatorname{In}_{2} \mathrm{O}_{3}$ physical constants, we find that acoustic phonon modes dominate over optical and piezoelectric modes. Then, according to the result of Bardeen and Shockley,[22] the phonon-limited mobility can be approximated as:

$$
\begin{aligned}
& \mu_{p h}(T)=A * T^{-3 / 2}, \\
& \text { where } \mathrm{A}=\frac{(8 \pi)^{1 / 2} e \hbar^{4} c_{i i}}{3 E_{1}^{2} m^{* 5 / 2} k_{B}^{3 / 2}}
\end{aligned}
$$


Grain boundary-affected performance of $\mathrm{Zn}$ and In oxide transparent conductors

and $\hbar=h / 2 \pi$ is the reduced Planck's constant, $c_{i i}$ is the elastic constant in direction of phonon propagation, and $E_{l}$ is a parameter with units of energy related to the magnitude of energy band shifts upon physical deformation of the crystalline lattice.

Under the working approximation that grain boundary and phonon scattering act independently, Equations 1 and 2 can then be combined using Matthisen's rule to yield the following simple model for temperature-dependent carrier mobility:

$$
\mu_{\text {Hall }}(T)^{-1} \approx \mu_{g b}(T)^{-1}+\mu_{p h}(T)^{-1} .
$$

This model can be expected to describe the higher temperature portion of the experimental data, where thermionic transport dominates over the thermally-assisted quantum mechanical tunneling of carriers through barriers. Indeed, the mobility data collected at above $\sim 115 \mathrm{~K}$ for both indium and zinc oxides can be well accounted for using this approach (Figure 5, Table 1).

The visibly different shapes of the carrier mobility $v s$. temperature curves for indium oxide and zinc oxide are largely accounted for by differences in $V_{b}$ across the two sets of samples, as calculated by fitting the model in Equation 3 to the portion of the experimental datasets collected above $115 \mathrm{~K}$. The indium oxide $V_{b}$ values range from $6.4-9.8 \mathrm{meV}$ and are notably smaller and less variable than the analogous values for $\mathrm{ZnO}$. For zinc oxide, $V_{b}$ increases markedly from $23.2 \mathrm{meV}$ for the sample deposited at $417^{\circ} \mathrm{C}$ to $37.3 \mathrm{meV}$ for the sample deposited at $334{ }^{\circ} \mathrm{C}$. These trends also neatly correspond with the observed room-temperature electrical transport data presented in Figures 1 and 3, which show an approximately constant $\operatorname{In}_{2} \mathrm{O}_{3}$ conductivity across the range of deposition temperatures and a lower overall $\mathrm{ZnO}$ conductivity that is highly sensitive to film deposition temperature.

The $L$ parameter, or effective grain size, varies only slightly across all samples except for the $\mathrm{ZnO}$ film deposited at $334{ }^{\circ} \mathrm{C}$. The much lower value of $L$ for this sample may suggest that structural changes within the grains of this sample may be occurring in addition to the grain boundary effects that are of primary interest here. Finally, the best fit $A$ values are also presented for reference. $A$ is a function of several correlated physical parameters $\left(c_{i i}\right.$ and $\left.E_{l}\right)$ that cannot be independently determined from these data alone. In any case, $A$ does not change dramatically for any of the samples studied and will thus not be further discussed here. 


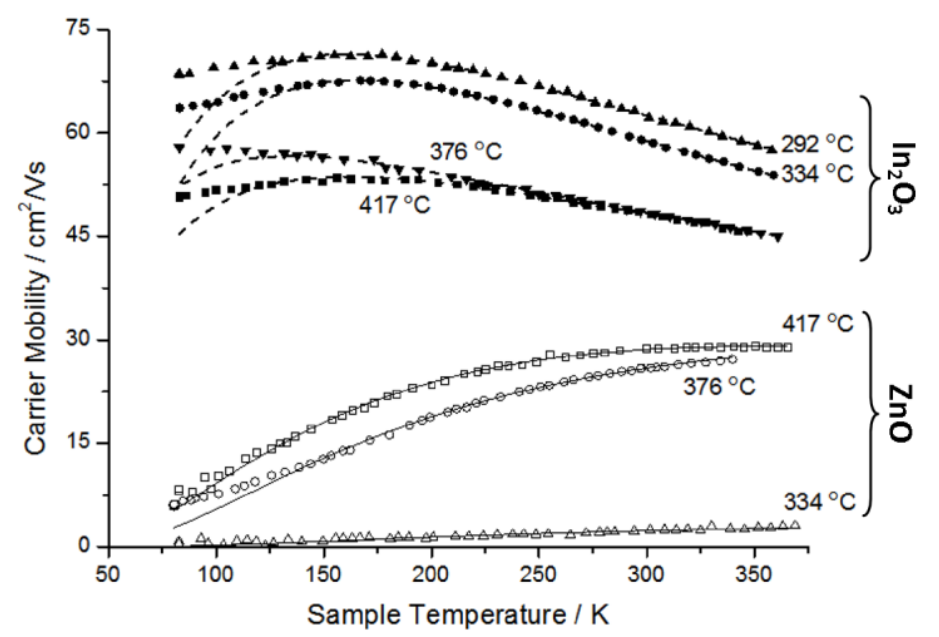

Figure 5. Hall carrier mobility as a function of temperature for $\mathrm{ZnO}$ (open symbols) and $\operatorname{In}_{2} \mathrm{O}_{3}$ (closed symbols) thin film samples prepared by spray pyrolysis at the indicated deposition temperatures. The parameters of the model described in the main text are optimized to produce the best fit line associated with each data set.

Table 1. Best fit parameter values of the model (Equation 3) used to describe the variable temperature carrier mobility data from each sample in Figure 5.

\begin{tabular}{ccccc}
\hline & $T_{\text {dep }}\left({ }^{\circ} \mathrm{C}\right)$ & $A\left(10^{6} \mathrm{~cm}^{2} \mathrm{~K}^{3 / 2} / \mathrm{Vs}\right)$ & $L(\mathrm{~nm})$ & $V_{b}(\mathrm{meV})$ \\
\hline \multirow{4}{*}{$\mathbf{I n}_{\mathbf{2}} \mathbf{O}_{\mathbf{3}}$} & 417 & 2.8 & 3.8 & 7.8 \\
& 376 & 1.3 & 5.9 & 9.8 \\
& 334 & 1.8 & 5.6 & 8.8 \\
& 292 & 3.0 & 3.6 & 6.4 \\
\hline \multirow{3}{*}{$\mathbf{Z n O}$} & 417 & 1.4 & 4.1 & 23.2 \\
& 376 & 2.4 & 4.4 & 28.6 \\
& 334 & 2.1 & 0.52 & 37.3 \\
\hline
\end{tabular}

\subsection{Photoconductivity response}

Further insight into the causes for and differences between these potential barriers can be found by considering the photoconductive behaviour of these samples. It is well established that $\mathrm{ZnO}$ exhibits a phenomenon known as "persistent photoconductivity," wherein the conductivity of a sample changes upon exposure to light with energy greater than that of the $\mathrm{ZnO}$ bandgap. This irradiation induces the desorption of a variety of air-derived, oxygen-containing species that are adsorbed at the grain boundaries, which reduces the height of the potential barriers there and temporarily increases the electrical conductivity.[23, 24] If the irradiation stops with the sample still exposed to air, the conductivity will eventually returns to its original state as readsorption of atmospheric species leads to the regeneration of grain boundary acceptor states.[25] The effect is called "persistent" because it depends on the relatively slow chemical kinetics of desorption and adsorption rather than being a purely electronic process; because of this, the changes in conductivity can 


\section{Grain boundary-affected performance of $\mathrm{Zn}$ and In oxide transparent conductors}

persist for hours or even days after the removal of the light source before completely decaying back to its original, dark value.

In addition to being of fundamental interest, we propose that this effect is a useful tool for assessing the extent to which the conductivity in a TCO film is affected by interaction of its grain boundaries with adsorbed species from the atmosphere. Because the net effect of irradiation is to clean the grain boundaries of labile acceptor defects, the larger the conductivity change upon such treatment, the more limiting such species are to electrical conductivity in the dark state for that sample. Figure 6 shows the relative photoconductive response upon exposure of $\mathrm{ZnO}$ and $\operatorname{In}_{2} \mathrm{O}_{3}$ samples prepared at various deposition temperatures to an $8 \mathrm{~W} \mathrm{UV}$ lamp with emission centred at $365 \mathrm{~nm}$. All of the samples show some degree of persistent photoconductivity, implying that similar physical processes are occurring in both $\mathrm{ZnO}$ and $\operatorname{In}_{2} \mathrm{O}_{3}$.

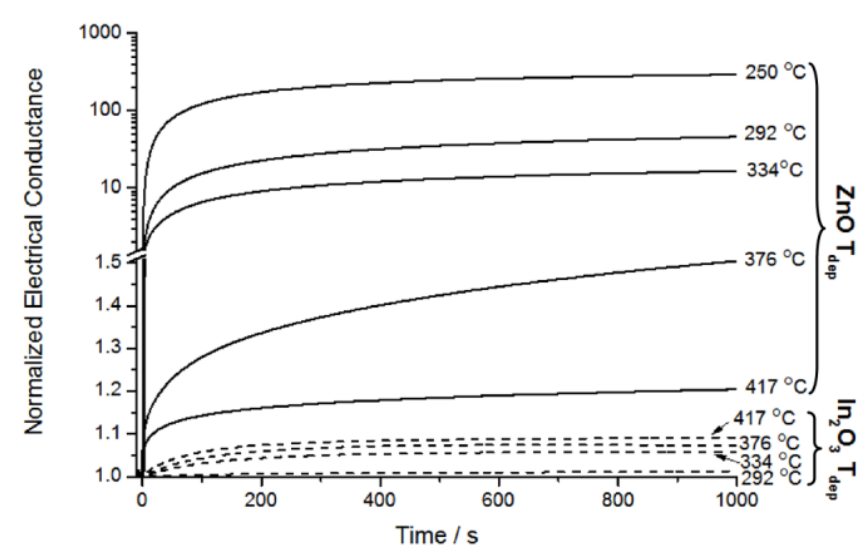

Figure 6. Normalized photoconductive response of zinc oxide (solid lines) and indium oxide (dashed lines) thin films deposited at the indicated deposition temperatures $\left(T_{d e p}\right)$. Note the break where the $\mathrm{y}$-axis scale changes from linear to logarithmic. $\mathrm{T}=0$ is defined as the time when the lamp is switched on, and all conductance values are normalized to their corresponding $\mathrm{T}=0$ value.

\subsection{Rationalizing indium and zinc oxide performance}

Turning to the question of performance differences between zinc and indium oxide, Figure 6 shows that all $\mathrm{In}_{2} \mathrm{O}_{3}$ samples show a substantially smaller conductivity increase upon irradiation than even the least responsive $\mathrm{ZnO}$, that deposited at the highest $\underline{T}_{\text {dep }}$. This indicates that the performance of the indium oxide thin film samples is significantly less limited by grain boundary acceptor states formed upon interaction of the sample with the air. Temperature-programmed desorption has previously been used to show that surface hydroxyls desorb from $\mathrm{ZnO}$ in two distinct peaks which occur at approximately $220{ }^{\circ} \mathrm{C}$ and $270{ }^{\circ} \mathrm{C}$.[26] Analogous experiments performed on thin ITO films showed water evolution likewise occurs in 2 steps. However, these processes occur at the lower temperatures of $150-165{ }^{\circ} \mathrm{C}$ and $~ 242{ }^{\circ} \mathrm{C}$.[27] Similar experiments performed on indium oxide films likewise report hydroxide desorption temperatures less than 
Grain boundary-affected performance of $\mathrm{Zn}$ and In oxide transparent conductors

$200{ }^{\circ} \mathrm{C}$. $[28,29]$ The higher desorption temperatures for $\mathrm{ZnO}$ imply a stronger bond between the oxide surface and water, and thus a greater tendency for the dissociative chemisorption of atmospheric moisture and the attendant creation of conductivity limiting potential barriers. Beyond just water, both experiment and calculation have shown that many substances interact strongly $\mathrm{ZnO}$ surfaces in a way that leads to the formation of surface acceptor states, a feature that has made $\mathrm{ZnO}$ very valuable for catalytic applications, but in light of our analysis, appears to be detrimental to its performance as a TCO material.[30-32]

These conclusions should certainly not be taken to suggest that indium oxide is entirely immune from the effect of acceptors at grain boundaries. In fact, it is undoubtedly possible to prepare indium oxide samples with low conductivity and a large photoconductive response. However, to do so appears to require rather strenuously oxidizing conditions, for example sputtering deposition using a pure oxygen atmosphere[33] or post-deposition treatment with ozone.[34] These are conditions which far exceed those necessary to produce marked reductions in the conductivity of $\mathrm{ZnO}$, for which we have found spray pyrolysis deposition in air instead of nitrogen is enough to result in 2 or more orders of magnitude electrical conductivity decrease. We propose that such differences in the surface reactivity of $\operatorname{In}_{2} \mathrm{O}_{3}$ and $\mathrm{ZnO}$ to moisture and oxygen can explain indium oxide's superior overall electrical conductivity, its relative insensitivity to (non-extreme) deposition conditions, and the excellent environmental stability that makes it the TCO of choice for many applications.

In addition to intrinsic differences in the energetics of adsorption onto $\mathrm{ZnO}$ and $\operatorname{In}_{2} \mathrm{O}_{3}$ surfaces, the influence of morphology and porosity on the accessibility and sensitivity of intergrain contacts to environmental contaminants should not be overlooked. Yamazoe, et al. provide a useful general discussion on this topic.[35] Figure 6 shows also that the magnitude of photoresponse differs dramatically among the $\mathrm{ZnO}$ samples as a function of deposition temperature and that the lower deposition temperature $\mathrm{ZnO}$ samples suffer substantially more from the effect of air-derived species adsorbed at grain boundaries than the samples prepared at higher $T_{d e p}$. We surmise that this could at least partially be due to differences in gross film morphology as a function of deposition temperature, whereby the more porous, feather-like structure of the low $T_{d e p}$ film allows more ready exposure of the grain surfaces to the environment than the stacked hexagonal platelets of films deposited at $376^{\circ} \mathrm{C}$ and $417^{\circ} \mathrm{C}$ (Figure 4).

Interestingly, the temperature dependence of indium oxide photoconductivity magnitude is reversed from that of zinc oxide, in that the $\operatorname{In}_{2} \mathrm{O}_{3}$ sample deposited at $292{ }^{\circ} \mathrm{C}$ shows the smallest change and the sample deposited at $417^{\circ} \mathrm{C}$ shows the largest. Why this should be the case is not immediately obvious from 


\section{Grain boundary-affected performance of $\mathrm{Zn}$ and In oxide transparent conductors}

the transport modelling summarized in Figure 5 and Table 1, which shows that these samples should possess very similar average grain boundary barrier heights. However, as before, for this series $\operatorname{of} \operatorname{In}_{2} \mathrm{O}_{3}$ thin films, at least a qualitative correlation can be made with the surface morphologies observed using SEM (Figure 4). The $\mathrm{In}_{2} \mathrm{O}_{3}$ samples deposited at $250{ }^{\circ} \mathrm{C}$ and $292{ }^{\circ} \mathrm{C}$ consist of crystalline grains which appear to be intimately joined. As the deposition temperature increases, the grains become larger and possess more distinct boundaries, which may facilitate the ingress/egress of atmospheric species. Additionally, the tendency towards higher crystallinity leads to an increased surface roughness that exposes a greater depth of the thin film sample to air.

We have also found that the magnitude of the photoconductive response in $\mathrm{ZnO}$ films deposited at $334{ }^{\circ} \mathrm{C}$ can be reduced by more than a factor of 6 when the film thickness is increased from $300 \mathrm{~nm}$ to 1000 $\mathrm{nm}$ (Figure 7). Given that these films are likely composed of materials with similar intrinsic properties, owing to the same chemistry and deposition temperature used, the more limited access of atmosphere into the interior of a thicker sample is a straightforward explanation for the difference in behaviour. Additionally, the broadly similar timescales on which the photoconductive effects in these 2 samples saturate is not consistent with an effect acting through the different full depths of both samples, but instead suggests that significant changes due to irradiation are occurring in a limited (likely near-surface) volume that is similar in both samples.

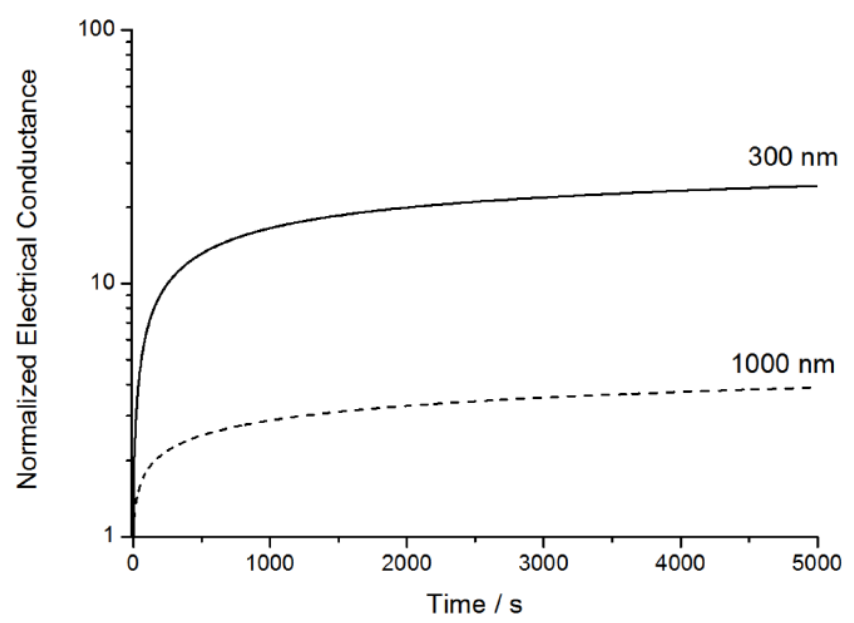

Figure 7. Normalized photoconductive response of zinc oxide samples of $1000 \mathrm{~nm}$ (dashed line) and $300 \mathrm{~nm}$ (solid line) thickness deposited at $334{ }^{\circ} \mathrm{C}$.

Taken together, these two concepts of differing intrinsic surface reactivity and the influence of film morphology have significant power to rationalize the major performance trends that have been seen in TCO research. For instance, the similar electrical properties of $\mathrm{ZnO}$ and $\mathrm{In}_{2} \mathrm{O}_{3}$ single crystals noted earlier can be explained by the fact that conduction in this case is dominated by the electronic transport properties within the 


\section{Grain boundary-affected performance of $\mathrm{Zn}$ and In oxide transparent conductors}

bulk. In contrast, with thin films (and especially polycrystalline thin films) surface and grain boundary effects can potentially become highly significant. While fully admitting that a great variety of morphologies can be achieved even using one deposition technique, Figure 8 provides one example of the morphological differences between $\mathrm{ZnO}$ thin film samples prepared using different techniques by our group. Vacuum-based physical deposition techniques such as pulsed laser deposition and magnetron sputtering tend to produce films with dense, relatively smooth surface morphologies lacking clearly defined access pathways into the film interior. It should then be no surprise that the highest conductivity $\mathrm{ZnO}$ and doped $\mathrm{ZnO}$ are invariably produced using such techniques. On the other hand, solution-based deposition techniques, like the spray pyrolysis used here, tend to produce surface morphologies with clearly defined grains and grain surfaces, the formation of which is facilitated by solvent- and thermally-assisted mass transport on the heated substrate surface. With such morphologies, a reasonable assumption would be that grain boundary access in solutionprocessed films is easier than with the vacuum-deposited samples, thus allowing the surface sensitivity of $\mathrm{ZnO}$ to have a larger effect on conductivity. This explains the progressive decrease in the electrical performance of vacuum- and solution-processed $\mathrm{ZnO}$ thin films from that of the ideal, single-crystal case. Looked at from the opposite angle, the striking insensitivity of indium oxide surfaces to environmental influence means, importantly, that top-performing $\operatorname{In}_{2} \mathrm{O}_{3}$ and ITO can be prepared by using any, and all, of these various deposition techniques.
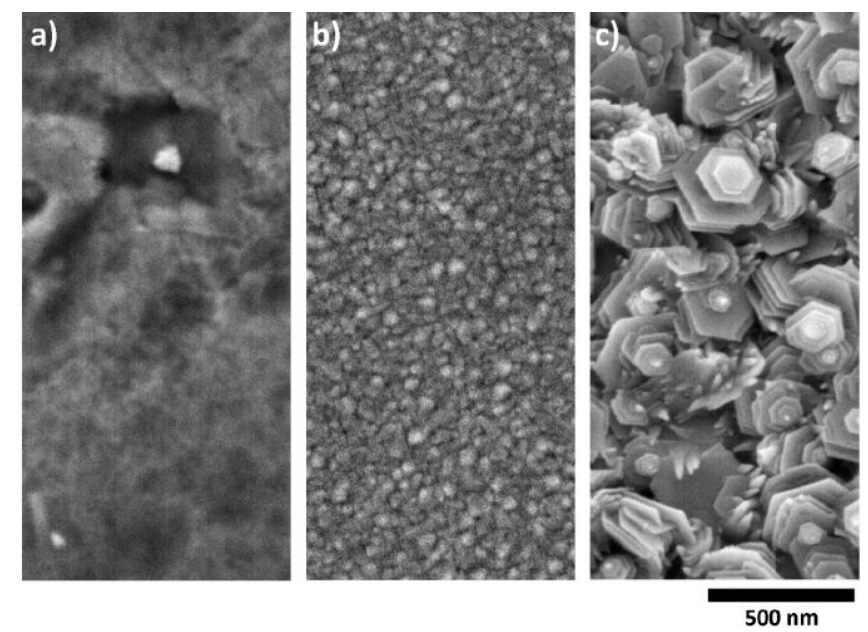

Figure 8. Comparison of morphologies of zinc oxide samples deposited using (a) pulsed-laser deposition, (b) magnetron sputtering, and (c) spray pyrolysis.

The implication is that understanding and ultimately modifying the morphology and surface chemistry of thin film TCO samples is of utmost importance in ongoing efforts to improve TCO performance. For example, Hüpkes, et al. have reported that annealing of doped zinc oxides at $650{ }^{\circ} \mathrm{C}$ for 24 hours using a 
Grain boundary-affected performance of $\mathrm{Zn}$ and In oxide transparent conductors

capping layer of amorphous $\mathrm{Si}$ to prevent film evaporation led to substantial improvements in carrier mobility.[36] In later work, they describe a corresponding increase in environmental stability and ascribe these effects to grain boundary reconstructions that restrict the diffusion of ambient species into the interior of the film.[37] Admittedly, this is a rather harsh treatment that would be difficult to scale up in its current form. Nevertheless, this is a very interesting proof-of-principle, and development of milder methods to modify the morphology of $\mathrm{ZnO}$ (whether before or after deposition) in a way that limits access of atmospheric species may well be of substantial practical use. A related alternative is to consider encapsulation of the types that are currently under extensive research, say, for OLED devices with oxidation- or moisture-sensitive active layers.[38, 39] Passivation of $\mathrm{ZnO}$ or doped $\mathrm{ZnO}$ surfaces using agents such as organic polymers,[40, 41] barrier films,[42] inorganic oxides or nitrides,[43-45] or thiocyanates[46] have been reported for $\mathrm{ZnO}$ materials intended other uses, but not yet been extensively examined for $\mathrm{ZnO}$ in TCO applications.

\section{Conclusion}

This study emphasizes the importance of carefully considering and interrogating grain boundary chemistry when assessing the conductivity and environmental stability of transparent conducting oxides. This is of particular relevance in efforts to replace the incumbent $\operatorname{In}_{2} \mathrm{O}_{3}$-based TCOs with lower cost alternatives, such as those based on $\mathrm{ZnO}$. The quantitative and qualitative assessments performed here of the grain boundary potential barriers that form due to atmospheric exposure can explain both the limited maximum conductivity of $\mathrm{ZnO}$ and the substantial decline in $\mathrm{ZnO}$ performance with decreasing deposition temperature. This same analysis performed on a comparable series of $\operatorname{In}_{2} \mathrm{O}_{3}$ thin film samples shows the remarkable resistance of this TCO material to the development of grain boundary potential barriers, and hence performance degradation caused by exposure to suboptimal environmental conditions, either during or after deposition. Indeed, the outstanding insensitivity of $\operatorname{In}_{2} \mathrm{O}_{3}$ to these deleterious effects as compared to $\mathrm{ZnO}$ throws the challenge of replacing the former with the latter into stark focus.

While these results do not imply that grain boundary effects are the only important factors limiting the performance of TCOs, it does show that any efforts to improve the performance of TCO materials in general, and of $\mathrm{ZnO}$ in particular, must necessarily account for and deal with their influence. Even more broadly, we argue that the best chance of making progress in this field lies in understanding and specifically addressing the physical factors (certainly including grain boundary potential barriers) that limit the electrical performance of real materials. Towards this end, we believe that developing methods to modify the structure or chemistry of 
Grain boundary-affected performance of $\mathrm{Zn}$ and In oxide transparent conductors

grain boundaries will greatly assist in the development of ITO alternatives, and hope that the concepts and ideas presented here will encourage the growth of fundamentals-based approaches to TCO research going forward.

\section{Acknowledgements}

The authors gratefully acknowledge financial support from the Clarendon Scholarship Fund, The Queen's College (Oxford), and an EPSRC IAA Award. We are also most thankful to Prof. Jon Dilworth for many useful discussions and assistance in the preparation of this manuscript.

\section{References}

[1] Ginley D, Hosono H and Paine D C 2011 Handbook of Transparent Conductors (New York: Springer)

[2] Kumar A, Zhou C 2010 The race to replace tin-doped indium oxide: Which material will win? ACS Nano 411

[3] Ellmer K 2012 Past achievements and future challenges in the development of optically transparent electrodes Nat. Photonics 6809

[4] Minami T, Miyata T, Ohtani Y, Kuboi T 2007 Effect of thickness on the stability of transparent conducting impurity-doped $\mathrm{ZnO}$ thin films in a high humidity environment phys. status solidi RRL 1 R31

[5] Theelen M, Dasgupta S, Vroon Z, Kniknie B, Barreau N, van Berkum J, Zeman M 2014 Influence of the atmospheric species water, oxygen, nitrogen and carbon dioxide on the degradation of aluminum doped zinc oxide layers Thin Solid Films $\mathbf{5 6 5} 149$

[6] Lin W, Ma R X, Xue J, Kang B 2007 RF magnetron sputtered ZnO:Al thin films on glass substrates: A study of damp heat stability on their optical and electrical properties Sol. Energy Mater. Sol. Cells 911902

[7] Greiner D, Gledhill S E, Köble C, Krammer J, Klenk R 2011 Damp heat stability of Al-doped zinc oxide films on smooth and rough substrates Thin Solid Films $\mathbf{5 2 0} 1285$

[8] Minami T 2005 Transparent conducting oxide semiconductors for transparent electrodes Semicond. Sci. Technol. 20 S35

[9] Patil P S 1999 Versatility of chemical spray pyrolysis technique Mater. Chem. Phys. 59185

[10] Vai A T, Kuznetsov V L, Jain H, Slocombe D, Rashidi N, Pepper M, Edwards P P 2014 The transition to the metallic state in polycrystalline n-type doped $\mathrm{ZnO}$ thin films Z. Anorg. Allg. Chem.

6401054 
Grain boundary-affected performance of $\mathrm{Zn}$ and In oxide transparent conductors

[11] Vai A T, Kuznetsov V L, Dilworth J R, Edwards P P 2014 UV-induced improvement in ZnO thin film conductivity: A new in situ approach J. Mater. Chem. C 29643

[12] Ellmer K, Mientus R 2008 Carrier transport in polycrystalline transparent conductive oxides: A comparative study of zinc oxide and indium oxide Thin Solid Films $\mathbf{5 1 6} 4620$

[13] Minami T 2008 Present status of transparent conducting oxide thin-film development for indium-tin-oxide (ITO) substitutes Thin Solid Films 5165822

[14] Benamar E, Rami M, Messaoudi C, Sayah D, Ennaoui A 1999 Structural, optical and electrical properties of indium tin oxide thin films prepared by spray pyrolysis Sol. Energy Mater. Sol. Cells $\mathbf{5 6}$ 125

[15] Swanepoel R 1983 Determination of the thickness and optical constants of amorphous silicon $J$. Phys. E: Sci. Instrum. 161214

[16] MacDonald W A 2004 Engineered films for display technologies J. Mater. Chem. 144

[17] Weiher R L 1962 Electrical properties of single crystals of indium oxide J. Appl. Phys. 332834

[18] Hutson A R 1957 Hall effect studies of doped zinc oxide single crystals Phys. Rev. 108222

[19] Shapira Y, McQuistan R B, Lichtman D 1977 Relationship between photodesorption and surface conductivity in $\mathrm{ZnO}$ Phys. Rev. B 152163

[20] Blatter G, Greuter F 1986 Carrier transport through grain boundaries in semiconductors Phys. Rev. B 333952

[21] Seto J Y W 1975 The electrical properties of polycrystalline silicon films J. Appl. Phys. 465247

[22] Bardeen J, Shockley W 1950 Deformation potentials and mobilities in non-polar crystals Phys. Rev. 8072

[23] Mollwo E 1954 Photoconductivity Conference (New York: John Wiley and Sons)

[24] Melnick D A 1957 Zinc oxide photoconduction, an oxygen adsorption process J. Chem. Phys. 261136

[25] Heiland G 1961 Photoconductivity of zinc oxide as a surface phenomenon J. Phys. Chem. Solids 22227

[26] Morishige K, Kittaka S, Moriyasu T, Morimoto T 1980 Thermal desorption study of surface hydroxyls on ZnO J. Chem. Soc., Faraday Trans. 176738

[27] Sawada Y, Seki S, Sano M, Miyabayashi N, Ninomiya K, Iwasawa A, Tsugoshi T, Ozao R, Nishimoto Y 2004 Evolution of water vapor from indium-tin-oxide transparent conducting films fabricated by dip coating process J. Therm. Anal. Calorim. 77751

[28] Barraud L, Holman Z C, Badel N, Reiss P, Descoeudres A, Battaglia C, De Wolf S, Ballif C 2013 Hydrogen-doped indium oxide/indium tin oxide bilayers for high-efficiency silicon heterojunction solar cells Sol. Energy Mater. Sol. Cells 115151 
Grain boundary-affected performance of $\mathrm{Zn}$ and In oxide transparent conductors

[29] Koida T, Shibata H, Kondo M, Tsutsumi K, Sakaguchi A, Suzuki M, Fujiwara H 2012

Correlation between oxygen stoichiometry, structure, and opto-electrical properties in amorphous $\mathrm{In}_{2} \mathrm{O}_{3}: \mathrm{H}$ films J. Appl. Phys. 111063721

[30] Au C T, Hirsch W, Hirschwald W 1988 Adsorption and interaction of carbon dioxide, formic acid and hydrogen/carbon dioxide mixtures on (1010) zinc oxide surfaces studied by photoelectron spectroscopy (XPS and UPS) Surf. Sci. 199507

[31] Wöll C 2007 The chemistry and physics of zinc oxide surfaces Prog. Surf. Sci. 8255

[32] Dutta G, Sokol A A, Catlow C R A, Keal T W, Sherwood P 2012 Activation of carbon dioxide over zinc oxide by localised electrons ChemPhysChem 133453

[33] Bender M, Katsarakis N, Gagaoudakis E, Hourdakis E, Douloufakis E, Cimalla V, Kiriakidis G 2001 Dependence of the photoreduction and oxidation behavior of indium oxide films on substrate temperature and film thickness J. Appl. Phys. 905382

[34] Xirouchaki C, Kiriakidis G, Pedersen T F, Fritzsche H 1996 Photoreduction and oxidation of asdeposited microcrystalline indium oxide J. Appl. Phys. 799349

[35] Xu C, Tamaki J, Miura N, Yamazoe N 1991 Grain size effects on gas sensitivity of porous $\mathrm{SnO}_{2}$-based elements Sens. Actuators, B 3147

[36] Warzecha M, Owen J I, Wimmer M, Ruske F, Hotovy J, Hüpkes J 2012 High mobility annealing of transparent conductive oxides IOP Conf. Ser.: Mater. Sci. Eng. 34012004

[37] Hüpkes J, Owen J I, Wimmer M, Ruske F, Greiner D, Klenk R, Zastrow U, Hotovy J 2014 Damp heat stable doped zinc oxide films Thin Solid Films $\mathbf{5 5 5} 48$

[38] Park J S, Chae H, Chung H K, Lee S I 2011 Thin film encapsulation for flexible AM-OLED: A review Semicond. Sci. Technol. 26034001

[39] Yang Y Q, Duan Y 2014 Optimization of $\mathrm{Al}_{2} \mathrm{O}_{3}$ films deposited by $\mathrm{ALD}$ at low temperatures for OLED encapsulation J. Phys. Chem. C 11818783

[40] Qin L, Shing C, Sawyer S, Dutta P S 2011 Enhanced ultraviolet sensitivity of zinc oxide nanoparticle photoconductors by surface passivation Opt. Mater. 33359

[41] Xu X, Feng L, He S, Jin Y, Guo X 2012 Solution-processed zinc oxide thin-film transistors with a low-temperature polymer passivation layer IEEE Electron Device Lett. 331420

[42] Nagamoto K, Hara T, Sakuma H, Ishii K 2014 Highly moisture resistant IGZO films with a barrier layer for flexible transparent conductive substrates CPMT Symposium Japan (ICSJ) 27

[43] Schmidt O, Geis A, Kiesel P, Van de Walle C G, Johnson N M, Bakin A, Waag A, Döhler G H 2006 Analysis of a conducting channel at the native zinc oxide surface Superlattices Microstruct. 39 8

[44] Hong D, Wager J F 2005 Passivation of zinc-tin-oxide thin-film transistors J. Vac. Sci.

Technol., B 23 L25 
Grain boundary-affected performance of $\mathrm{Zn}$ and In oxide transparent conductors

[45] Seo H, Bae J, Kim D, Park Y, Kim C, Kang I B, Chung I, Choi J, Myoung J 2009 Reliable bottom gate amorphous indium-gallium-zinc oxide thin-film transistors with $\mathrm{TiO}_{\mathrm{x}}$ passivation layer Electrochem. Solid-State Lett. 12 H348

[46] Jalali N, Briscoe J, Woolliams P, Stewart M, Weaver P M, Cain M, Dunn S 2013 Passivation of zinc oxide nanowires for improved piezoelectric energy harvesting devices J. Phys.: Conf. Ser. 476 012131 